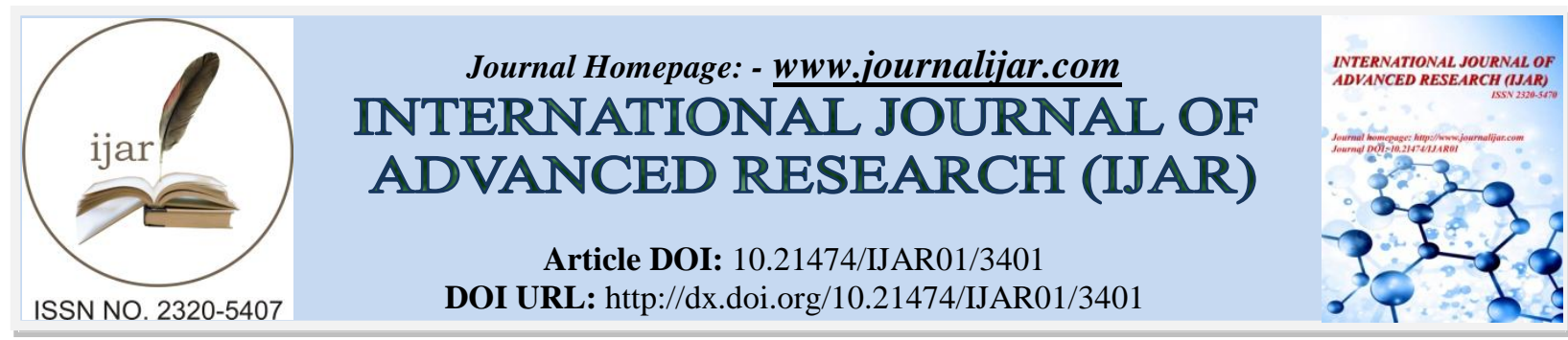

RESEARCH ARTICLE

\title{
COMPARATIVE STUDY OF INTRAOPERATIVE COMPLICATIONS IN PSEUDOEXFOLIATION SYNDROME WITH NORMAL PATIENTS IN CATARACT SURGERY.
}

Dr. D.Y.Patil Hospital \& Research Institute.

\section{Manuscript Info}

Manuscript History

Received: 20 December 2016

Final Accepted: 15 January 2017

Published: February 2017

Key words:-

Pseudoexfoliation syndrome, Manual

Small Incision Cataract Surgery,

Intraoperative Complications and

Postoperative Complications.

\section{Abstract}

Background And Objective: Pseudoexfoliation syndrome is an agerelated systemic diseasemanifesting itself primarily in the eyes which is characterized by the accumulation of microscopic granular amyloidlike protein fibres. Studies have shown that these patients have higher rates of intraoperative complications compared to the patients without the condition. This study was undertaken to determine and compare the intraoperative complications in Pseudoexfoliation patients with Normal patients in cataract surgery.

Methods: It's a hospital based Prospective observational study of 100 patients, 50 patients having Pseudoexfoliation syndrome and 50 patients without Pseudoexfoliation syndrome attending department of Ophthalmology, D. Y. Patil hospital and Research Institute, Kolhapur, between May 2014-April 2016.

Results:The average age of patients was 66 years, with male predominance and with a higher incidence of bilateral involvement. 13 (26\%) patients with Pseudoexfoliation syndrome had intraoperative complications while $4(8 \%)$ patients without Pseudoexfoliation syndrome had intraoperative complication. Among all complications, the incidence among patients with Pseudoexfoliation syndrome and patients without Pseudoexfoliation syndrome were 6(12\%) and 2(4\%) for Zonular dehiscence, 4(8\%) and 1(2\%) patient for Posterior Capsular Rent and 3(6\%) and 1(2\%) patient for Vitreous loss respectively.

Conclusion: Inadequate mydriasis is one of the major preoperative complication with Pseudoexfoliation syndrome which has a bearing on the intraoperative complications. With proper preparation, Small Incision Cataract Surgery can be performed in this group of patients. In conclusion, with appropriate preoperative, intraoperative and postoperative care the risks of complications can be minimized and favorable outcomes can be achieved in cataract surgery with Pseudoexfoliation syndrome.

Copy Right, IJAR, 2017,. All rights reserved.

\section{Introduction:-}

Pseudoexfoliation Syndrome is an age related generalized disorder involving abnormal production or turnover of extra-cellular matrix in ocular tissues, orbital tissues, skin and visceral organs. The exact etiopathogenesis of this condition and chemical composition of the material still remains unknown. 
In the eye, Pseudoexfoliation syndrome is characterized clinically by small white deposits of material in the anterior segment, most commonly in the pupillary border and the anterior lens capsule. The most consistent diagnostic feature is three distinct zones of Pseudoexfoliation material seen on the lens capsule after full dilatation.

Additional subtle clinical signs that help in early diagnosis are loss of pigment from peri-pupillary area producing trans illumination defects, insufficient mydriasis, and pigment dispersion into anterior chamber after mydriasis, deposition of melanin over trabecular meshwork and Schwalbe's line. The existence of posterior synechiae without any other cause and hemorrhage in the iris stroma after mydriasis are also suggestive of Pseudoexfoliation syndrome. Deposition of material on the zonular fibres weakens it leading to Phacodonesis, subluxation and dislocation of lens. The presence of secondary open angle glaucoma is known as glaucoma capsulare. This glaucoma has more serious clinical course and worse prognosis than primary open angle glaucoma, often not responding to medical therapy and requiring early surgical intervention. An increased incidence of nuclear cataract is seen. Pseudoexfoliation syndrome frequently goes undiagnosed leading to unexpected problems in management and during surgery.Therefore, making the diagnosis often requires a careful slit-lamp examination after pupillary dilatation.

Due to involvement of virtually all structures by Pseudoexfoliation material, patients have a significantly greater risk for a variety of complications during cataract surgery. Poor mydriasis, pigment dispersion, combined with Phacodonesis and zonular dialysis predisposes to posterior capsular rupture and vitreous loss. Intra operative modifications like sphincterotomy can lead to post-operative irregular pupil and iris pigment dispersion.

Possible pre-operative, intra-operative and post-operative measures to avoid or minimize these complications include an increased awareness of Pseudoexfoliation syndrome, a careful slit lamp examination after full pupillary dilatation, adequate control of intra- ocular pressure pre-operatively, avoidance of iris manipulation, adequate pupillary dilatation, use of heparin coated intra-ocular lenses, use of capsular tension ring in selected cases and judicious use of steroids post- operatively.

\section{Materials and Methods:-}

A total number of 100 cases (50 patients with Pseudoexfoliation and 50 normal patients as control) attending D. Y. Patil Hospital Kadamwadi Kolhapur fulfilling the inclusion and exclusion criteria will be studied. Inclusion criteria followed were: 1)Patients 50 years and above of age belonging to either sex diagnosed to have cataract with Pseudoexfoliation on the basis of slit lamp examination before and after pupillary dilatation.2)Patients above 50 years of age belonging to either sex diagnosed to have cataract without Pseudoexfoliation on the basis of slit lamp examination before and after pupillary dilatation.Exclusion criteria for the study was: 1) Patients less than 50 years of age.2) Patients with traumatic cataract.3) Patients with history of exposure to intense infrared lights i.e. glass blowing.4) Patients with eye diseases other than Pseudoexfoliation or early cataract. 5)Patients with Diabetes Mellitus.

Pre-operative evaluation was done which included: 1)Visual acuity testing for distance and near using Snellen's distant chart and near vision chart respectively.2)Refraction and correction where required.3)External ocular examination.4)Slit lamp bio microscopic examination for evidence of the following findings : Pseudoexfoliation material in the pupillary margins,Morphological alterations of the cornea, Anterior chamber depth and pigment dispersion in the anterior chamber, Iridodonesis, Presence of posterior synaechiae, Zones of Pseudoexfoliation on the anterior surface of the lens capsule, Phacodonesis or frank subluxation/dislocation of lens, Measurement of pupil size before and after dilatation of pupil and Pupillary reactions. 5)Intraocular pressure using Goldmann tonometer. 6)Gonioscopy with Goldmann three mirror lens in all patients with Pseudoexfoliationsyndrome. 7)The pupils were then dilated with a combination of $10 \%$ phenylephrine and tropicamide $1 \%, 1$ drop was instilled every 5 minutes over a 15 minute interval. 8)This was followed by slit lamp examination for measuring pupil dilatation, examination of lens capsule for central and peripheral zones of Pseudoexfoliation material deposition and evaluation of lens for the type of cataract.9)Fundoscopy10)Lacrimal patency test 11)Keratometry 12)A-scan and Intraocular lens power calculation by SRK-2 formula.

Other investigations included urine examination for detection of sugar and albumin. All patients were given systemic antibiotics (tablet ciprofloxacin $500 \mathrm{mg}$ b.d.) on the preoperative day. On the day of surgery pupils were dilated adequately using instillation of $1 \%$ tropicamide and $10 \%$ phenylephrine eye drops every 10 minutes before surgery. Selected Patients who had high intraocular pressure were given intravenous mannitol $20 \%$ one gram per 
kilogram bodyweight and oral Acetazolamide 250 milligram 2 times a day depending on level of intraocular pressure preoperative day and also 1 hour prior to surgery.

\section{Results:-}

In this study of 100 cases, we studied many different parameters in patients with Pseudoexfoliation syndrome and control group.The age distribution (as seen in Table 1) shows $6(12 \%)$ patients of age group $50-59$ years, 37(74\%) patients of age group $60-70$ years and $7(14 \%)$ of age group $71-80 \%$. The average age of patients was 66.28 years and about $37(74 \%)$ of patients were above 60 years of age. $30(60 \%)$ were males and $20(40 \%)$ were females. 31 $(62 \%)$ of the patients had clinical bilateral involvement of Pseudoexfoliation syndrome and 19 (38\%) had unilateral involvement. $36(72 \%)$ of the patients had Pseudoexfoliative materials present on the pupillary margin, 9 (18\%) of the patients had pseudoexfoliative materials present on the iris surface, 2(4\%) of the patients had Iris Atrophy, $1(2 \%)$ of the patient had Iridodonesis and $2(4 \%)$ of the patients had posterior synechiae in this study group.

Out of the 50 patients, 43(86\%) of the patients had open angles and 7(14\%) of the patients had narrow angles as compared to patients without Pseudoexfoliation syndrome, 49 (98\%) had open angles and 1(2\%) patient had occluded angle.18(60\%) had peripheral zone, 12(40\%) had both peripheral zone and centralzone and none of them had only centralzone. 19(38\%) of the patients had Mature Cataract and 31(62\%) of them had Immature cataract with Pseudoexfoliation syndrome compared to $16(32 \%)$ of the patients had Mature Cataract and 34(68\%) of the patients had immature cataract without Pseudoexfoliation syndrome

The intraocular pressure distribution in patients with and without Pseudoexfoliation syndrome. 28 (56\%) of the patients had sufficient mydriasis, and 22(44\%) of the patients had insufficient mydriasis as compared to patients without Pseudoexfoliation syndrome $48(96 \%)$ of patients had sufficient mydriasis, and 2(4\%) of the patients had insufficient mydriasis(as seen in Table $2 \mathrm{a}$ and b).13 (26\%) of the patients with Pseudoexfoliation syndrome had intraoperative complication while $37(74 \%)$ patients did not have any complications. $4(8 \%)$ of the patients without Pseudoexfoliation syndrome had intraoperative complication while 46 (96\%) patients did not have any complications. (as seen in Table 3a and b) While comparing the intraoperative complications in patients with and without Pseudoexfoliation syndrome it shows statistically significant difference $(\mathrm{p}<0.05)$.

As seen in table 4, 6(12\%) of patients had Zonular dehiscence while this complication was seen in 2(4\%) patients without Pseudoexfoliation syndrome, $4(8 \%)$ of the patients had Posterior Capsular Rent while 1(2\%) patient without Pseudoexfoliation syndrome had this complication and $3(6 \%)$ of the patients had Vitreous loss while $1(2 \%)$ patient without Pseudoexfoliation syndrome had this complication.

All patients were implanted with intraocular lens after employment of various surgical modifications like, Sphincterotomy, Synecholysis and Posterior Capsular Ring.Out of the total 50 patients, 8(26.67\%) patients who had one intraoperative complications, $5(62.5 \%)$ of them had insufficient mydriasis and $3(37.5 \%)$ of the patients had adequate mydriasis. 6 patients $(12 \%)$ had sphincterotomy, 5 patients(10\%) had synechiolysis and $2(4 \%)$ underwent both during cataract surgery. 2(4\%) patients had capsular tension ring.

Table 1:- Age Distribution In Patients With Psedoexfoliation Syndrome ( $\mathrm{N}=50)$.

\begin{tabular}{|l|l|l|}
\hline Age & Number Of Patients & Percentage \\
\hline $50-59$ YEARS & 6 & 12 \\
\hline $60-70$ YEARS & 37 & 74 \\
\hline $71-80$ YEARS & 7 & 14 \\
\hline TOTAL & 50 & 100 \\
\hline
\end{tabular}

Table 2a:- Intraocular Pressure In Patients With Pseudoexfoliation Syndrome (N = 50)

\begin{tabular}{|c|c|c|}
\hline Iop & Number Of Patients & Percentage \\
\hline $10-20 \mathrm{~mm} \mathrm{Hg}$ & 37 & 74 \\
\hline $21-30 \mathrm{Mm} \mathrm{Hg}$ & 12 & 24 \\
\hline$>30 \mathrm{~mm} \mathrm{Hg}$ & 1 & 2 \\
\hline Total & 50 & 100 \\
\hline
\end{tabular}


Table 2b:- Intraocular Pressure In Patients Without Pseudoexfoliation Syndrome $(\mathrm{N}=50)$

\begin{tabular}{|c|c|c|}
\hline Iop & Number Of Patients & Percentage \\
\hline $10-20 \mathrm{~mm}$ of $\mathrm{Hg}$ & 46 & 62 \\
\hline $21-30 \mathrm{~mm}$ of $\mathrm{Hg}$ & 3 & 2 \\
\hline$>30 \mathrm{~mm}$ of $\mathrm{Hg}$ & 1 & 100 \\
\hline Total & 50 & 6 \\
\hline
\end{tabular}

Table 3 A:- Intraoperative Complications In Pseudoexfoliation Syndrome ( $=50)$

\begin{tabular}{|c|c|c|}
\hline Complications & Number Of Patients & Percentage \\
\hline Occurred & 13 & 26 \\
\hline Not Occurred & 37 & 74 \\
\hline Total & 50 & 100 \\
\hline
\end{tabular}

Table 3 B:- Intraoperative Complications In Patients Without Pseudoexfoliation Syndrome $(\mathrm{N}=50)$

\begin{tabular}{|c|c|c|}
\hline Complications & Number Of Patients & Percentage \\
\hline Occurred & 4 & 8 \\
\hline Not Occurred & 46 & 92 \\
\hline Total & 50 & 100 \\
\hline
\end{tabular}

Table 4:- Intraoperative Complications In Patients With And Without Pseudoexfoliation Syndrome

\begin{tabular}{|c|c|c|}
\hline Complications & $\begin{array}{c}\text { Number Of Patients Having } \\
\text { Pseudoexfoliation Syndrome }\end{array}$ & $\begin{array}{c}\text { Number Of Patients Without } \\
\text { Pseudoexfoliation Syndrome }\end{array}$ \\
\hline Zonular Dehiscence & 6 & 2 \\
\hline Posterior Capsular Rupture & 4 & 1 \\
\hline Vitreous Loss & 3 & 1 \\
\hline
\end{tabular}

\section{Discussion:-}

This study consisted of 50 eyes of 50 patients with Pseudoexfoliation syndrome and 50 eyes of 50 patients without Pseudoexfoliation syndrome who underwent manual small incision cataract surgery. The average age of patients was 66.28 years and about 37 (74\%) of patients were above 60 years of age.Pseudoexfoliation syndrome usually occurs between 60 to $80 \mathrm{yrs}$, the average age being $70 \mathrm{yrs}$. Studies regarding the sex distribution of Pseudoexfoliation syndrome are conflicting. Women have predominated in some series while other studies have found equal or greater prevalence in men. In this study, males were more affected than females.31 (62\%) of the patients had clinical bilateral involvement of Pseudoexfoliation syndrome and 19 (38\%) had unilateral involvement. A review of literature comparing the frequency of monocular versus binocular involvement in various series is not conclusive. ${ }^{1}$

$36(72 \%)$ of the patients had Pseudoexfoliative materials present on the pupillary margin, $9(18 \%)$ of the patients had pseudoexfoliative materials present on the iris surface, 2(4\%) of the patients had Iris Atrophy, 1(2\%) of the patient had Iridodonesis and 2 (4\%)of the patients had posterior synechiae in this study group. A study by Ritch Schlotzer. Scherhardt 2,3 (2001) stated that deposits of Pseudoexfoliation material on the iris sphincter and pupillary margin are seen in $84 \%$ patients. Thus, the most prominent and consistent clinical finding is the Pseudoexfoliation material at the pupillary border. $20(40 \%)$ had peripheral zone, $30(60 \%)$ had both peripheral zone and central zone and none of them had only central zone. The peripheral zone of pseudoexfoliation material is a consistent finding and the central zone is not always apparent. ${ }^{4}$ Tarkkanen found the central zone absent in $18 \%$ of cases in his study while Ritch, Schlotzer - Scherhardt found it absent in 20-60\% of their cases. 9(30\%) of the patients had Mature Cataract and $21\left(70 \%\right.$ ) of them had Immature cataract. ${ }^{5,6}$ All of them, i.e. $100 \%$, had Nuclear Cataracts. Cortical Cataract was present along with advanced nuclear cataract and none of the patients had isolated cortical cataract. Seland et al have reported a higher incidence of nuclear cataract in eyes with pseudoexfoliation syndrome with fewer cortical cataracts. ${ }^{7}$ Hietanen $\mathrm{J}$ et al have also reported nuclear cataract to be the predominant type of cataract in Pseudoexfoliation syndrome.

Out of 50 patients in the present study group 37 (74\%) of the patients had IOP in the range $10-20 \mathrm{~mm} \mathrm{Hg}, 12(24 \%)$ of the patients in the range of $21-30 \mathrm{~mm} \mathrm{Hg}$ and $1(2 \%)$ of the patient had IOP more than $30 \mathrm{~mm} \mathrm{Hg}$ as compared to 
50 patients without Pseudoexfoliation syndrome, 46(92\%) of the patients had IOP in the range 10- $20 \mathrm{~mm} \mathrm{Hg}$, $3(6 \%)$ of the patients in the range of 21-30 mm Hg and $1(2 \%)$ of the patient had IOP more than $30 \mathrm{~mm} \mathrm{Hg}$. Overall 17 patients who had raised IOP were managed by either by giving intravenous Mannitol $20 \%$ one gram/kilogram body weight over a period of 30 minutes to one hour, along with oral acetazolamide 250 milligrams twice a day on preoperative day and intravenous Mannitol $20 \%$ one gram/kilogram body weight given one hour prior to surgery or only oral acetazolamide 250 milligrams twice a day for 3 days.

In other studies, with pseudoexfoliation syndrome, 20\% had glaucoma and increased IOP at the time of diagnosis, as compared to our study where $17 \%$ of patients had raised IOP. This underscores the need for careful follow-up in patients who have pseudoexfoliation syndrome. Pseudoexfoliation syndrome accounts for $15-20 \%$ of cases of open angle glaucoma.

$1(2 \%)$ of the patients had iridodonesis. This is because the iris in Pseudoexfoliation syndrome is more rigid due to vascular compromise and various other changes like deposition of Pseudoexfoliation material, Atrophy, Loss of iris stroma.

As part of management of rigid undilating pupil, 6 patients (12\%) had sphincterotomy, 5 patients (10\%) had synechiolysis and $2(4 \%)$ underwent both during cataract surgery. $2(4 \%)$ patients had capsular tension ring. Alfaite et al in their study of 31 eyes of Pseudoexfoliation syndrome undergoing ECCE noted a statistically significant increase ( $\mathrm{p}$ value $<0.01$ ) in the need to perform sphincterotomies. Kuchle et al noted $3.4 \%$ of their 76 patients to require surgical Synechiolysis and/or mechanical dilatation of pupil intraoperatively. ${ }^{13}$

Amongst 50 patientswith Pseudoexfoliation syndrome 13(26\%) had intraoperative complication while 37 (74\%) patients did not have any complications compared to $4(8 \%)$ of the patients without Pseudoexfoliation syndrome had intraoperative complication while $46(96 \%)$ patients did not have any complications. 6(12\%) of patients had Zonular dehiscence while this complication was seen in 2(4\%) patients without Pseudoexfoliation syndrome, 4(8\%) of the patients had Posterior Capsular Rent while 1(2\%) patient without Pseudoexfoliation syndrome had this complication and $3(6 \%)$ of the patients had Vitreous loss while 1(2\%) patient without Pseudoexfoliation syndrome had this complication.

\section{Conclusion:-}

Small incision cataract surgery in eyes with Pseudoexfoliation syndrome is associated with an increased incidence of complications both preoperatively and intraoperatively. Inadequate mydriasis is one of the major preoperative complications in eyes with Pseudoexfoliation syndrome which has a bearing on the intraoperative complications like posterior capsular rent and vitreous loss. Adequate surgical modifications such as Sphincterotomy and/or Synechiolysis in these eyes with inadequate mydriasis reduce the intra operative complications. These pupil enlargement procedures are advocated during cataract surgery.

All though cataract surgery in Pseudoexfoliation syndrome is challenging, if the surgeon is aware of the condition preoperatively and pays meticulous attention to the surgical technique, a good outcome can be expected.

\section{Bibliography:-}

1. Hammer, Schlotzer- Schrehardt, Naumann. "Unilateral or Asymmetric Pseudoexfoliation syndrome? An electron microscopic study”., Klin Monatsbl Augenheilkd 2001; 216(6): 388- 392.

2. Schlotzer-Schrehardt et al. "pseudoexfoliation syndrome -ocular manifestation of a systemic disorder?" Archives of ophthalmology, 1992; 110(12) 1752-1756.

3. Asano N, Schlotze - Scherhardt, Naumann GO. "A histo-pathological study of iris changes in Pseudoexfoliation", Ophthalmology, 1996; 102: 1279 - 1290.

4. M. Bruce Shield's Text book of Glaucoma, 5th edition, Lippincott Williams \& Wilkins, Philadelphia.

5. Tarkkanen A, Forsius H, eds. Exfoliation syndrome. Acta Ophthalmol 1988; 66(suppl. 184): 1.

6. Schlotzer - Schrehardt U, Naumann G O, Kuchle M. Pseudoexfoliation syndrome for the comprehensive ophthalmologist. Intraocular and systemic manifestations. Ophthalmology, 1998; 105: 951-68.

7. Seland GH and Chylack LT Jr. "Cataracts in the Exfoliation Syndrome (Fibrilliopathia epitheliocapsularis)",, Transophthalmol Soc U.K, 1982; 102: 375. 
8. Sunde OA. Senile exfoliation of the anterior lens capsule. Acta Ophthalmol, 1956; 45: 1.

9. Wishart PK, Spathe GL and Poryzees EM. "Anterior Chamber angle in the Exfoliation Syndrome", British Journal of Ophthalmology, 1985; 69: 103.

10. Futa R, Furnyoshi N, Shimizu T. "Clinical features of capsular glaucoma in comparision with primary open angle glaucoma in Japan”. Acta Ophthal 1992; 70: 214-219.

11. Moreno M.J., Duch S and Lajara. "pseudoexfoliation syndrome:Clinical factors related to capsular rupture in cataract surgery.”. Acta Ophthal (Copenh) 1993; 71: 181 - 184.

12. Ritch Schlotzer, Scherhardt et al. "Unilateral or Asymmetric Pseudoexfoliation syndrome, An Ultra structural Study", Achieves of Ophthalmology, 2001; 119: 1023 - 1031.

13. Kuchle et al. "Anterior chamber depth and complications during cataract surgery in eyes with pseudoexfoliation syndrome." American journal of ophthalmology 2000; 129: 281- 285. 